\title{
Polysèmes
}

Revue d'études intertextuelles et intermédiales

\section{Fascination de la miniature : Steven Millhauser, entre théorie et fiction}

\section{Anne Ullmo}

\section{OpenEdition}

\section{Journals}

Édition électronique

URL : http://journals.openedition.org/polysemes/684

DOI : 10.4000/polysemes.684

ISSN : 2496-4212

Éditeur

SAIT

Édition imprimée

Date de publication : 1 janvier 2012

Pagination : 249-266

ISSN : 0999-4203

\section{Référence électronique}

Anne Ullmo, «Fascination de la miniature : Steven Millhauser, entre théorie et fiction », Polysèmes [En ligne], 12 | 2012, mis en ligne le 01 mars 2015, consulté le 02 mai 2019. URL : http:// journals.openedition.org/polysemes/684 ; DOI : 10.4000/polysemes.684

Ce document a été généré automatiquement le 2 mai 2019.

Polysèmes 


\title{
Fascination de la miniature : Steven Millhauser, entre théorie et fiction
}

\author{
Anne Ullmo
}

1 «The realm of the miniature awaits its passionate and scholarly explorer » constate Steven Millhauser, à l'ouverture de l'essai «The Fascination of the miniature", qu'il consacre précisément à la miniature ${ }^{1}$, impliquant sans doute que ce royaume a enfin trouvé son explorateur ; et par son ambiguïté grammaticale, le titre de cette réflexion "The Fascination of the Miniature», laisse entrevoir les potentialités réflexives de cet objet d'art dont on peut certes scruter les détails minuscules mais qui, dans un retournement fantastique, peut tout aussi bien vous happer par son regard, à l'instar des miroirs qui, chez Millhauser, retiennent les belles dames dans la captivité de leur regard irrésistible ${ }^{2}$. De cette polysémie, qui motive également le titre de Georges Didi-Huberman Ce que nous voyons, ce qui nous regarde ${ }^{3}$, Steven Millhauser tire un parti poétique en particulier dans des textes courts qui précèdent ou suivent, selon les cas, l'exploration théorique de 1983 sur la miniature.

2 Je souhaiterais par conséquent m'interroger sur les altérations que subit la miniature comme objet et comme concept, sur la transposition de l'un vers l'autre, selon un va-etvient qui laisse entendre que Millhauser se méfie tout particulièrement de la théorie et plus encore de la critique. L'inclusion de cette forme d'art dans un récit pose d'autre part la question des relations inter-sémiotiques et de l'utilisation figurale d'un objet que Philippe Hamon qualifierait peut-être de « technème ».

\section{Miniature et distorsion}

Dans "The fascination of the Miniature ", Steven Millhauser étudie la miniature sous l'angle de l'écart, condition sine qua non de l'enchantement du spectateur. La petite taille ne suffit pas; il faut y ajouter la notion d'échelle et de relation avec un objet à l'aune duquel peut se mesurer sa version miniaturisée: "The miniature, then, must not be confused with the merely minute. For the miniature does not exist in isolation: it is by 
nature a smaller version of something else. The miniature, that is to say, implies a relation, a discrepancy $»^{4}$.

Mais le simple décalage, l'écart, quoique conditions nécessaires, ne seraient pas non plus tout à fait suffisants pour que s'émerveille le regard enfantin de l'observateur. Car enfin, le gigantisme pourrait, lui aussi, s'y retrouver. La clé de la fascination réside alors dans la dimension affective de la miniature, liée à celle de distorsion : «Unlike the gigantic, the miniature is without dread. Here lies part of its secret charm. We allow ourselves to surrender completely, untroubled by danger $\aleph^{5}$. Le charme discret de la miniature dépendrait, par conséquent, de son aspect rassurant, réconfortant, parce que renvoyant à du connu.

5 L'auteur transpose sans cesse cette réflexion théorisée dans ses récits. Si l'on y rencontre le gigantisme des parcs d'attractions ou des grands magasins qui, en capturant les badauds, se substituent au monde qu'ils dupliquent dans les moindres détails, on y découvre aussi quelques petites compositions, plus stupéfiantes encore que ces rêves prométhéens car non moins rigoureuses dans leur reproduction du réel; cependant, au sentiment de crainte qui règne dans les récits de gigantisme, à l'égarement dans les méandres de ces labyrinthes borgésiens, se substitue alors le ravissement du tout petit. Les taxinomies interminables, propres à cette « rhétorique de l'excès " ${ }^{6}$ que l'auteur met au service de l'infiniment grand, font alors place à une expression plus subtile de la passion démiurgique. Car les architectures pléthoriques n'ont pas l'apanage de ce désir de se mesurer à Dieu. Si Millhauser abandonne un temps les listes à l'ampleur whitmanienne, où la parataxe remplace la structure séquentielle, dans un souffle "démocratique », c'est parce que la miniature, art délicat s'il en est, est un style tout autant qu'un objet; et parce qu'elle invite à la possession, elle ne témoigne pas moins de ce « refus de n'être pas Dieu »; " As a child I longed to have an extra pair of eyes at the back of my head. What was that longing but an impatience with human limitation, a reluctance not to see everything, a refusal, in short, not to be God? »" "Voir tout", "montrer tout", sont une façon de contenter celui qui, en chacun de nous, réclame sa part de détails, de minutie, de précision, de mimétique poussé à l'extrême: à la frustration de l'enfant devant de médiocres reproductions d'arbres ou de bateaux, répond, par réaction, l'extrême précision des descriptions de l'auteur. La nouvelle "The Sepia Postcard ", extraite du recueil The Barnum Museum ${ }^{8}$, qui place au-devant de la scène une carte postale découverte dans un magasin poussiéreux, synthétise un grand nombre des intuitions de l'auteur sur le thème de la miniature, en introduisant, outre la notion de précision mimétique, l'antithèse animé / inanimé, que Millhauser développe par ailleurs dans nombre de ses récits d'automates.

\section{« The Sepia Postcard » ou la miniature animée}

6 D'un long discours didactique au cours duquel le narrateur de "The New Automaton Theater » met en lumière ce qu'il estime être les différences entre l'art de la miniature et l'art de l'automate, émerge l'idée que le premier serait un art sans vie: «the art of the miniature is in essence a lifeless art, an art of stillness $»^{9}$. Or, si les automates peuvent eux-mêmes être des créatures miniaturisées animées, la miniature peut à son tour être susceptible de mouvement, comme en témoigne le récit, à la frange du fantastique, de "The Sepia Postcard ». Le narrateur homodiégétique de ce récit est un citadin au bord de la crise de nerf, qui fuit la ville et ses tracas, ainsi qu'une relation de couple incertaine, 
pour s'immerger dans les brumes d'une petite station balnéaire de la côte Est du nom de Broome. La phrase inaugurale met cette sorte de journal intime sous le signe de la tension et du surmenage ( I was tense, irritable, overworked», 93), qui justifieront par la suite les étranges observations d'un narrateur, irritable comme peuvent l'être ceux de Poe.

7 Le temps est hivernal et les pluies répétées forcent le personnage au confinement dans une chambre d'hôtel mal chauffée. L'achat d'une carte postale surannée chez un bouquiniste sera le point de départ d'une mise en récit de l'image. S'intercalant entre les différentes étapes du compte-rendu des quelques heures passées dans l'hôtel du bord de mer, les descriptions de la carte postale sont des récits en miniature, des touts autonomes dont les lectures consécutives permettent littéralement que s'anime la scène, comme c'est le cas pour le narrateur. En effet, à la faveur d'une observation de plus en plus attentive, le tableau devient séquence cinématographique, sa mise en mouvement observant les règles de la lanterne magique :

The melancholy brown photograph showed a rocky point extending into a lake or a river; on the other side of the water was a brown forest of pines, and above the trees were long thin brown clouds and a setting brown sun. In the upper left-hand corner of the sky, in small brown capital letters, was the single word INNISCARA. On the farthest rock two small figures were seated, a man and a woman, looking out at the water. Beside the man I could make out a straw hat and a walking stick. The woman was bareheaded, her hair full and tumbling. The details were difficult to distinguish in the dim light, but the very uncertainty seemed part of the romantic melancholy of the brown scene. (100)

8 Sorte de refrain dans la trame diégétique, cette description initiale de la carte postale sera déclinée six fois avec chaque fois d'infimes variations. Le lecteur se prend vite au jeu de ce travail minutieux de type "spot the differences » et doit juxtaposer les différentes descriptions (chacune étant un tout autonome dans l'économie de la nouvelle) de façon à pouvoir mesurer à son tour les écarts entre la première et la dernière version. Ce travail de découpage fait ici partie de l'expérience de lecture, comme si la mémoire ne suffisait pas à enregistrer ce qu'un œil chaque fois plus attentif décèle.

Ce fragment descriptif, qui s'insère visuellement dans le corps du texte comme une vignette, semble répondre à la nomenclature réaliste, avec son porte-regard et les verbes qui lui sont classiquement associés ("I could make out», "to distinguish»), son quadrillage topographique rationalisant ("on the other side", "above», "on the farthest rock ", «beside ») et l'exhaustivité recherchée par le regard de l'observateur qui va même jusqu'à mentionner les mots dans la représentation ("INNISCARA»), détail marquant la vraisemblance, effet de réel intriguant, présence typographique qui attire l'œil sur le signifiant plus que le signifié (Inniscara Lake, à quelques kilomètres de Cork en Irlande).

10 L'intention descriptive du narrateur est soulignée par la redondance des verbes de perception, volontairement simples: aux deux verbes marquant l'effort d'attention nécessaire pour apercevoir les détails minuscules du paysage et des personnages représentés sur la carte postale ( I I could make out» ou «the details were difficult to dinstinguish ", 100) répond l'éventail de plus en plus large du vocabulaire de la vue au fil des transformations ténues de l'expression du couple d'amoureux : les termes «I saw", "I could make out ", "I could see ", " to read her expression ", "I seemed to detect ", dans la deuxième étape de la transformation, sont repris à peine modifiés lors des étapes suivantes, la répétition des mêmes syntagmes opérant comme un écran neutre sur lequel peuvent se jouer les infimes variations de l'imperceptible métamorphose. 
11 L'oeil est ici un objectif à focale variable, capable de se resserrer avec une précision surnaturelle sur l'infime: un brin d'herbe, les traits d'une femme, des cils, des lèvres entrouvertes ou l'ombre d'une pommette. L'effet grossissant est poussé à l'extrême, jusqu'à l'absurde même, jusqu'à la décomposition fractale de ce monde en miniature : de réalistes, les détails deviennent hyperréalistes et font basculer le lecteur dans une autre dimension.

Le jeu des répétitions achève de transformer la description en une partition de l'étrange : répété six fois, l'adjectif «brown » met la scène sous le signe du sépia (dans d'autres nouvelles Millhauser décline les bleus et les blancs) et rythme le texte avec le systématisme d'un métronome. De sorte que la couleur se transforme progressivement en musique, avec, au fil des descriptions, le passage du « piano » («brown softness », 104) au «forte » (« the sharpness of the image was almost startling»,106)?

Sous le regard nerveux et inquiet du narrateur, la miniature perd de son caractère rassurant, tandis que la caméra interne du narrateur enchaîne les séquences les unes aux autres. Jean Rousset, dans son étude du roman Histoire de Claude Simon, évoque ce même phénomène en termes de "réalisme illusionniste $»^{10}$, de théâtralisation et de glissement dans la mobilité, donc dans le temps.

\section{Éloge de la lenteur}

Pas question, semble nous dire Millhauser, de céder ici à cette avidité de la connaissance que l'on sait inséparable de l'expérience de lecture. Pas question, par conséquent, de "sauter impunément » les descriptions comme Barthes nous enjoint de le faire au sujet du texte réaliste classique ${ }^{11}$. Les fictions de Millhauser sont un éloge de la lenteur, une invite à n'attendre du texte que le texte. Souvenons-nous avec Barthes que « ce qui arrive au langage n'arrive pas au discours $»^{12}$. En d'autres termes, survoler un texte contemporain fait encourir le risque qu'il n'arrive rien. Comment, dans ce travail sur l'infime, percevoir en effet les menues fluctuations d'une expression ou d'une ride sur l'eau, si l'on n'astreint pas l'œil à l'effort de tension auquel est soumis le regard du narrateur? Les termes "tension ", "taut », «tense » et leurs variantes, opèrent comme un métadiscours, indicateur à la fois de la rigueur ascétique de l'écriture même et de l'attention exigée du lecteur.

15 «I like the variety of invention within a convention of rigorous triteness » (95) avoue le narrateur, pour justifier son plaisir à contempler la vitrine d'un magasin de souvenirs. Et c'est bien de cette « rigoureuse platitude » que se nourrit le style de Millhauser, inventif à l'intérieur des conventions du réalisme. Une platitude transcendée par cet usage très particulier que fait Millhauser de la répétition et dont on pourrait dire qu'il ressortit à cet érotisme dont parle Barthes dans Le Plaisir du texte ${ }^{13}$ : "en somme, le mot peut être érotique à deux conditions opposées, toutes deux excessives : s'il est répété à outrance, ou au contraire s'il est inattendu ». Millhauser choisit clairement l'érotisme de la scansion.

\section{Cathay ou l'écrivain micrographe}

Parcourir un texte de Millhauser, c'est valider à chaque instant l'injonction de Barthes : "ne pas dévorer, ne pas avaler, mais brouter, tondre avec minutie ", être en somme des « lecteurs aristocratiques $»^{14}$. L'œuvre de Millhauser témoigne de cette exigence de lenteur 
invoquée à la fois par Barthes et par Jean-Pierre Richard, pour qui la lecture « relève plutôt d'une insistance, d'une lenteur, d'un vœu de myopie $»^{15}$. Steven Millhauser est lui aussi une sorte de "micrographe ", au sens où il fait du détail sa chose ${ }^{16}$ : ce terme est employé au sujet des réalistes qui, persuadés d'habiter le siècle de l'uniformité et de l'indiscernable, préconisaient, par réaction, l'usage de la plume comme scalpel mais aussi comme loupe, comme seul moyen d'échapper au retour unheimlich du même.

Dans une perspective décalée par rapport à celle des écrivains du dix-neuvième siècle, qui choisissaient de s'appuyer sur le détail afin de revendiquer leur place dans le siècle et de suppléer à la déflation sémiotique autant que sémantique (que soulignent Philippe Hamon, mais aussi Luc Rasson ${ }^{17}$ ), Millhauser fait du détail un tout autonome et non plus la saillie, l'érection accidentelle sur un fond de platitude ${ }^{18}$. La miniature remplit donc une fonction, non pas de fragment (on a vu que chaque «lecture » de la carte postale pouvait constituer un tout autonome dans " The Sepia Postcard») mais de tableau à part entière, le mouvement n'étant imprimé que par la juxtaposition des images (on retrouve ici l'idée de succession, de séquences, comme au cinéma) et est interprétable comme tel.

Mais Millhauser franchit une nouvelle frontière dans un texte que l'on peinerait à qualifier de « récit » et qui propose la miniature à la fois comme référent et comme mode opératoire. La nouvelle "Cathay $»^{19}$ se présente comme une succession de textes courts sur un thème central : le Palais de l'Empereur de Chine, en un siècle indéterminé. Chacun de ces textes descriptifs est surmonté d'un titre composé d'un groupe nominal (« singing birds ", « clouds ", « hourglasses ", « dragons »), titres qui seront pour ainsi dire dépliés de façon à en souligner les aspects essentiels, sans emphase, avec la limpidité d'un haïku japonais. C'est à un narrateur homodiégétique, dont on ignore l'identité (insider certes, mais sans situation précise dans l'échelle sociale ?) qu'appartient de décrire en quelques mots les nuages de Cathay, les clepsydres de Cathay, les concubines de Cathay: longues d'une phrase ou de plusieurs paragraphes, ces sortes de vignettes témoignent d'une sobriété orientale. Si en effet, le haïku, le renku et le tanka sont des formes poétiques japonaises (et non chinoises) qui répondent à des critères formels très précis ${ }^{20}$, on peut néanmoins sentir le souffle de ces formes traverser ce texte (mais aussi, à la lumière de ce texte, d'autres textes).

Le groupe nominal « The Emperor's blue horses in a field of white snow », qui tient lieu de développement du titre "Blue Horses », a du haïku la brièveté, l'élément météorologique ainsi que la dimension visuelle et concrète qui en fait une évocation à la fois de l'icimaintenant et de l'éternité. Mais la démarche tout entière qui préside, non seulement à "Cathay » mais fort probablement à la plupart des nouvelles de Millhauser, est celle d'un retour vers ce que Barthes appelle la "théorie libératrice du signifiant». Plus qu'une théorie, c'est d'une pratique qu'il s'agit, dans le cas de Millhauser, qui nomme sans métaphore, reprend les mêmes signifiants encore et encore jusqu'à ce que l'impression devienne non plus visuelle mais sonore.

Ici, l'empire est un monde, un univers qui contient tout, y compris le langage dans sa totalité. Ainsi, chaque nuage porte un nom spécifique selon la forme qui est la sienne, un nom assorti d'un numéro qui, en se rapportant à un objet connu, en permettra la classification : "the name is always of an object, natural or artificial, that exists in our empire, which is so vast that it is said to contain all things " (148). On remarquera le rapport ironique au réalisme, un réalisme qui, ici, s'hypertrophie pour rejoindre les contrées borgésiennes d'une bibliothèque de Babel aux teintes de lapis lazuli. 
21 Si l'univers signifié est le grand Tout, les textes qui le représentent, bien que se succédant sous la forme d'une taxinomie, ne sont qu'au nombre de vingt et un. Pas de performance encyclopédique à la manière de Whitman; la dimension holistique n'est assurée que par la reprise de mêmes lexèmes d'une section à l'autre : employés sous la forme de titres, ils apparaîtront également en amont ou en aval dans une section dont ils ne sont pas les sujets principaux, comme c'est le cas d'un dictionnaire dont chaque entrée renvoie, par hypertexte, à une autre entrée, comme si chaque unité renvoyait à la totalité ; ces échos internes transforment le texte lui-même en macrocosme microscopique, en miniature de monde. Un monde dont les espaces entre les sections reproduisent les vides internes, les zones de rien qui permettent au lecteur de circuler librement de l'une à l'autre et de réinventer chaque fois son propre tableau de l'empire chinois.

Loin d'un quelconque souffle lyrique, "Cathay » en général et la section "Eyelids » en particulier, propose au contraire une vision de l'art sans artifice littéraire (que seraient la rime ou la métaphore), instaure l'apparente banalité comme événement textuel, une certaine légèreté humoristique venant même désamorcer tout pathos. L'usage du présent met ici en avant la combinaison de l'immuable et de l'éphémère. Ainsi, les groupes nominaux qui s'enchaînent sur un mode paratactique lors de l'évocation des différents types de paysages que recèlent les paupières des courtisanes ont eux-mêmes la force visuelle d'un haiku : " a tall willow with dripping branches; an arched bridge in snow; a pear blossom and hummingbird; a crane among cocks; rice leaves bending in the wind; a wall with open gate, through which can be seen a distant village on a hillside » (153). Ekphrasis miniatures, ces groupes nominaux purement dénotatifs s'imposent comme des tableaux générateurs de sensations, de réminiscences, peut-être, et semblent bruire (un bruissement de la langue sans la gangue des commentaires implicites ?) dans la plus pure limpidité de l'image.

Tout autant qu'au mode poétique japonais, la présente section fait songer à la technique $\mathrm{du}$ «blason », cette esthétique maniériste popularisée par Marot au XVI ${ }^{\mathrm{e}}$ siècle - Marot, qui déclencha en effet avec son épigramme du «Beau tétin », l'engouement pour ce jeu littéraire. Le blason est un court poème à la louange d'un objet dont on célèbre les vertus singulières. Le lyonnais Maurice Scève (1500-1562) s'est en particulier illustré dans ce genre en remportant le concours de blasons organisé par Marot en 1536, pour lequel il avait proposé cinq blasons : "Le sourcil », "La larme », "La gorge », " Le front ", " Le soupir ». Millhauser connaît très bien les littératures du Moyen-Âge et de la Renaissance pour s'être engagé dans un doctorat à l'université de Brown, et la délicatesse de la section consacrée aux paupières des courtisanes n'a rien à envier à celle qui caractérise les meilleurs de ces blasons français.

Le terme de "préciosité » aide à saisir cette indicible tension entre l'érotisme qui sourd dans les textes de Millhauser et une certaine forme d'idéalisation: l'expression «ambiguous messages» (152) au début de "Eyelids» traduit ce mouvement contradictoire d'appel et de mise à distance tout à la fois. De la même manière, on ne sait si c'est la miniature comme œuvre d'art à la surface de la paupière ou le regard de la courtisane aux profondeurs insoupçonnées qui fascine l'amant: "One can never be certain, when one sees a handsome courtier gazing passionately into the eyes of a beautiful lady, whether he is searching for the soul behind her eyes or whether he is striving to attain a glimpse of her elegant and dangerous eyelids » (152).

Simple donnée diégétique ou commentaire métatextuel ? L'ambiguïté règne en maître là aussi. Car ce que suggère ce texte, c'est qu'à la tentation des profondeurs partagée par les 
lecteurs du roman réaliste succède le regard innocent mais insistant du lecteur contemporain: plus que des herméneutes, les courtisans de Cathay sont de tendres voyeurs, des amoureux du signe, pour ce qu'il suggère et laisse entrevoir de beauté à venir. Il ne s'agit pas ici de décrypter les infimes lignes des tatouages miniaturisés mais simplement de parvenir, dans un clignotement de l'œil, à les voir: les «tantalizing glimpses » ou la « rapturous contemplation » (153) sont autant de promesses d'un plaisir moins charnel que spirituel.

Millhauser met ici en place un thème que l'on retrouvera dans les œuvres ultérieures, celui de l'entre-deux, du seuil entre le visible et l'invisible, " half-exposed and always hiding"; et il fait de cet interstice le lieu même de l'événement. Entre pureté et débauche, le visage de la courtisane, marqué à l'encre de chine, est un oxymore vivant. La nudité elle-même est moins érotique que ce paradoxe ; la découverte du détail sur le sein de la femme aimée procure une extase qui se passe de mots ( it is impossible to express the troubled excitement [...]», 153) parce que l'on est dans un mode de l'intime qui est de l'ordre du pulsionnel et si l'on en croit Freud, de la récompense.

\section{Possession et miniature}

Si le détail est la récompense donnée au micrographe, la miniature procède davantage d'un désir de maîtrise et de possession. Inversant l'adage bachelardien «je possède d'autant mieux le monde que je suis plus habile à le miniaturiser $»^{21}$, Millhauser fait de la miniature le point de départ d'une réflexion sur sa conception de l'art : « but not only is the miniature without dread, it also invites possession » (130).

Quelle est alors la place du référent dans cette démarche littéraire ? Les miniatures ne sont-elles pas auto-référentielles? Si le palais de l'Empereur contient son exacte reproduction sous une forme miniaturisée, qui en contient elle-même une autre, c'est peut-être bien parce que l'univers de Millhauser est fait d'emboîtements successifs et renvoie à une structure involuée qui ne réfère qu'à elle-même. On peut citer à ce propos un paragraphe dans lequel Rolf Stein donne des éléments d'analyse des jardins en miniature asiatiques :

La petitesse, loin d'être un élément accidentel des jardins en miniature, un pis-aller faute de place, donne au contraire plus de valeur à l'objet. En effet, plus la reproduction de l'objet naturel s'éloigne, par ses dimensions, de la réalité et plus il revêt un caractère magique ou mythique. Aménager un parc qui contient au complet toutes les essences et tous les êtres de l'univers, c'est déjà un acte magique (concentration de l'univers en son centre, la capitale, la demeure du roi). Mais le réduire en tout petit, le rendre maniable, accessible à un tour de main, c'est lui enlever le dernier semblant de réalité factice et l'élever au domaine de la seule réalité véritable : à l'espace mythique. L'instrument magique participe de la nature d'un artifice, l'artifice de celle d'un jouet. ${ }^{22}$

Le message est ici que la miniature allie subtilement les contraires, le phénoménal et le nouménal, la réalité sensible et la réalité intelligible ; Millhauser joue sur cette identité des contraires. Manipuler le monde est d'autre part une opération essentiellement ludique, elle est une jubilation pour l'artiste qui devient le puer lusor bachelardien qui s'amuse à posséder le monde comme un jouet ${ }^{23}$.

Il me semble que la réflexion que mène Millhauser sur la question de la miniature, amène à se poser une autre question, celle de sa relation à l'abstraction. En effet, si l'on peut dire que la théorie n'est jamais chez lui qu'une praxis (comme en témoigne finalement l'essai 
sur la miniature, que son style ne permet pas de différentier d'un texte fictionnel), cette pratique même incite à ne voir de l'œuvre que ce qu'elle veut bien montrer ${ }^{24}$ et l'on connaît le refus de Millhauser de se laisser capturer par le regard des critiques littéraires. Même si la miniature suppose un travail sur le détail, puisque Millhauser insiste sur la nécessité d'un détail d'autant plus fourni que l'objet est petit, et même si l'illusionnisme est l'un des paradigmes de ses œuvres, on ne peut éviter de percevoir la tentation du vide et de la tautologie chez cet auteur qui encourage l'immédiateté de la relation avec l'œuvre, débarrassée des écrans interprétatifs. Et s'il peut y avoir immédiateté, c'est aussi par ce que l'œuvre est conçue comme un tout, cette coexistence de toutes choses ensemble, cette gestalt (forme autonome, spécifique, immédiatement reconnaissable ${ }^{25}$ ) dont les parties sont si unifiées «qu'elles offrent un maximum de résistance à toute perception séparée $»^{26}$. On peut reprendre les propos du peintre Frank Stella au sujet de son art : « Ma peinture est basée sur le fait que seul s'y trouve ce qui peut y être vu. C'est réellement un objet [...] Si la peinture était assez incisive, assez précise, assez exacte, il vous suffirait simplement de la regarder. La seule chose que je souhaite que l'on tire de mes peintures et que j'en tire pour ma part, est que l'on puisse voir le tout sans confusion. Tout ce qui est à voir est ce que vous voyez $»^{27}$.

Qu'en est-il alors de la transposition de la théorie vers la pratique ? La théorie est une praxis pour Millhauser et la fiction n'oublie jamais le métadiscours ; chacun de ses textes, grand ou petit, roman ou nouvelle, est traversé par un impératif : donner à voir la magie de l'artifice.

\section{BIBLIOGRAPHIE}

\section{Euvres de Steven Millhauser}

“The Fascination of the miniature". Grand Street 2.4 (Summer 1983): 128-135.

“Cathay". The Penny Arcade. London: Phoenix, 1999, 147-164.

“The Sepia Postcard”. The Barnum Museum. New York: Poseidon Press, 1990, Dalkey Archive, 1997, 93-110.

\section{Ouvrages critiques}

Bachelard, Gaston. Études. Paris : Vrin, 1970.

Barthes, Roland. Le Plaisir du texte. Paris : Éditions du Seuil, 1973.

Chènetier, Marc. Steven Millhauser. Paris : Belin, 2003.

Cheng, François. Vide et plein. Paris : Éditions du Seuil, mai 1991.

Didi-Huberman, Georges. Ce que nous voyons, ce qui nous regarde. Paris : Éditions de Minuit, 1992.

Richard, Jean-Pierre. Microlectures. Paris : Éditions du Seuil, 1979.

Rousset, Jean. Passages, échanges et transpositions. Paris : José Corti, 1990. 
Rasson, Luc \& Schuerewegen, Franc (eds.). Pouvoirs de l'infime. Saint-Denis : PU de Vincennes, 1997.

Stein, Rolf A. Le Monde en petit. Paris : Idées et Recherches Flammarion, 1987.

\section{NOTES}

1. "The Fascination of the miniature", Grand Street 2.4 (Summer 1983): 128-135.

2. Steven Millhauser, "Cathay", In the Penny Arcade, 158.

3. Georges Didi-Huberman, Ce que nous voyons, ce qui nous regarde, Paris : Éditions de Minuit, 1992.

4. "The Fascination of the miniature", op. cit., 128-129.

5. Ibid., 130.

6. Marc Chènetier, Steven Millhauser, Paris : Belin, 2003, 79.

7. "The Fascination of the miniature", op. cit., 131.

8. The Barnum Museum, New York: Poseidon Press, 1990, Dalkey Archive, 1997. Les références entre parenthèses dans le texte ci-après renvoient à cette nouvelle.

9. “The New Automaton Theater", 91.

10. Jean Rousset, Passages, échanges et transpositions, Paris : José Corti, 1990, 160.

11. Roland Barthes, Le Plaisir du texte, Paris : Éditions du Seuil, 1973, 21 : « lisez lentement, lisez tout, d'un roman de Zola, le livre vous tombera des mains; lisez vite, par bribes, un texte moderne, ce texte devient opaque, forclos à votre plaisir : vous voulez qu'il arrive quelque chose, et il n'arrive rien; car ce qui arrive au langage n'arrive pas au discours [...] ».

12. Ibid.

13. Ibid., 58.

14. Ibid., 21.

15. Jean-Pierre Richard, Microlectures. Paris : Éditions du Seuil, 1979, 7.

16. Luc Rasson \& Franc Schuerewegen (eds.), Pouvoirs de l'infime, Saint-Denis : PU de Vincennes, 1997, 7.

17. «Autrefois, tout était en saillie, aujourd'hui, tout est en creux » affirme Félix Davin dans son «Introduction aux Études de mœurs au XIX ${ }^{e}$ siècle » de 1834. Ibid., 7

18. Bellemin-Noël, « L'infiniment détail », Pouvoirs de l'infime, op. cit., 24.

19. Steven Millhauser, "Cathay", The Penny Arcade, 147-164. Les références entre parenthèses dans le texte ci-après réfèrent à cette nouvelle.

20. Un haïku comporte 17 mores, découpage des sons plus fin que ne le permet la syllabe occidentale, en un tercet 5+7+5.

21. Gaston Bachelard, Études, Paris : Vrin, 1970, 142.

22. Rolf A. Stein, Le Monde en petit, Paris : Idées et Recherches Flammarion, 1987, 59-60.

23. Bachelard, op cit., 28.

24. Cf. la tautologie telle qu'elle est analysée par Georges Didi-Huberman dans Ce que nous voyons, ce qui nous regarde, op. cit., 32.

25. Cf. Didi-Huberman, ibid., 30.

26. R. Morris cité par Didi-Huberman, ibid., 31.

27. Ibid., 32. 
INDEX

oeuvrecitee Cathay, Sepia Postcard (The) 